\title{
Decreased Serum Total Bilirubin Level Predicts Early Neurological Deterioration in Patients with Acute Ischemic Stroke
}

\section{Xihua Sheng* \\ Huaping Du (D)* \\ Ying Tang}

Department of Neurology, Suzhou Ninth People's Hospital, Suzhou, Jiangsu, 215200, People's Republic of China

*These authors contributed equally to this work
Correspondence: Ying Tang

Department of Neurology, Suzhou Ninth People's Hospital, No. 2666, Ludang Road, Wujiang District, Suzhou, Jiangsu, 2I5000,

People's Republic of China

Tel +86 5I2-82885052

Email 158502I3093@I26.com
Objective: This study aimed to investigate the relationship between serum total bilirubin (TBil) level and early neurological deterioration (END) in patients with acute ischemic stroke of large artery atherosclerosis.

Patients and Methods: In this retrospective study, a total of 291 patients with acute ischemic stroke were enrolled. The demographic and laboratory dates were collected. Stroke severity had been assessed using the National Institutes of Health Stroke Scale (NIHSS). Multivariable logistic regression was used to examine the independent association between TBil and END.

Results: Approximately $63(21.6 \%)$ of the patients were diagnosed with END within the first seven days. The proportion of hypertension, diabetes mellitus (DM) and previous stroke/ transient ischemic attack (TIA) was significant greater in the lowest quartile $(<9.8 \mu \mathrm{mol} / \mathrm{l})$ of TBil. The proportion of patients with an elevated TBil levels was significantly lower in the END group than in the non-END group. After controlling for covariates, the first quartiles $(<9.8 \mu \mathrm{mol} / \mathrm{l})$ of TBil were still associated with END. In addition, an increased level of CRP and age were also associated with an increased risk of END.

Conclusion: The TBil levels in patients with acute cerebral infarction may be a useful biomarker for the prediction of END.

Keywords: acute ischemic stroke, early neurological deterioration, total bilirubin, predictor

\section{Introduction}

Early neurological deterioration (END) has been defined as any new neurological symptoms/signs or any neurological worsening. END occurs in 13-38\% of acute ischemic stroke cases and has been associated with reduced neurological function, increased fatality, and worse functional outcomes. ${ }^{1,2}$ Many studies have reported some biological or clinical factors that can predict END. ${ }^{3}$ However, many of these factors cannot explain the mechanisms of END.

Some studies have shown that bilirubin is a strong physiological antioxidant, and that low levels of bilirubin are closely related to atherosclerosis. ${ }^{4,5}$ The level of serum bilirubin in patients with acute ischemic cerebral infarction is lower than that in normal subjects and is related to the severity of stroke. ${ }^{6-8}$

However, the relationship between bilirubin and END is still unclear. Therefore, this study investigated the relationship between serum bilirubin level and END in patients with acute ischemic stroke. 


\section{Patients and Methods}

\section{Subjects}

This study was a single-center retrospective study. The patients were aged $\geq 18$ years and had developed acute ischemic stroke due to large artery atherosclerosis within $24 \mathrm{~h}$ of symptom onset presented to the stroke unit from January 2018 to June 2019. All patients underwent brain computed tomography (CT) scans to exclude cerebral or subarachnoid hemorrhage. Acute ischemic stroke was confirmed by brain magnetic resonance imaging (MRI) or brain CT within $24 \mathrm{~h}$ of admission. The main exclusion criteria were as follows: 1) probable stroke mimics, 2) some diseases might affected bilirubin excretion, such as infection, or inflammatory diseases, hematological diseases, hepatic and renal insufficiency. All patients received the best medical treatment. All patients or their legally authorized representatives provided written informed consent. This study was approved by the Ethical Committee of Suzhou Ninth People's Hospital (No. WJYY-201747).

\section{Data Collection}

The demographic data of the patients in the study such as age, sex, and vascular risk factors including hypertension, diabetes mellitus (DM), dyslipidemia, previous stroke or transient ischemic attack (TIA), coronary artery disease and atrial fibrillation were collected. All patients' venous blood samples were collected and analyzed following overnight fasting for at least $12 \mathrm{~h}$. Serum total bilirubin (TBil) and other biochemical dates were analyzed by a solid-phase chemiluminescent immunometric assay on Immulite 2000 (Siemens Healthineers, Erlangen, Germany) using the reagents provided by the manufacturer. The patients were divided into four groups based on the quartiles of serum TBil level.

\section{Neurological Severity and Early Neurological Deterioration}

We assessed stroke severity using the National Institutes of Health Stroke Scale (NIHSS). END was defined as follows: 1) an increment in the NIHSS score (consciousness and motor score are excluded) $\geq 2$ points; 2 ) an increment in the NIHSS consciousness score $\geq 1$ point; 3 ) an increment in the NIHSS score on motor of 1 or more points; or 4) any new neurological deficit during the first 7 days after symptom onset. ${ }^{9}$ We classified the patients into two groups according to the presence or absence of END.

\section{Statistical Analysis}

SPSS 19.0 (SPSS Inc., Chicago, IL, USA) was used for data analysis. Study participants were divided into four groups, based on quartiles of TBil levels. Continuous variables were expressed as mean \pm standard deviation (SD) or median (interquartile range [IQR]) and were compared using the analysis of variance, KruskalWallis test, or Wilcoxon rank-sum test, where appropriate. Categorical variables were expressed as frequency (\%) and compared using the chi-square test. Covariates with statistically significant differences $(P<0.05)$ were investigated using univariate analysis, and those with clinically important factors, even if they were not statistically significant, were adjusted for multivariate analysis. We analyzed the relationship between the level of TBil and END using the restricted cubic spline function with 3 knots at 10th, 50th and 90th percentiles of TBil. $P<0.05$ was considered statistically significant. ${ }^{10}$

\section{Results \\ Baseline Characteristics of the Study Subjects by Quartiles of Total Bilirubin}

A total of 291 patients with acute ischemic stroke were enrolled in this study. Their mean age was $69.4 \pm 12.6$ years, $160(55 \%)$ were male, and $63(21.6 \%)$ were diagnosed with END within the first 7 days (Table 1). The median TBil level was $12.3 \mu \mathrm{mol} / \mathrm{L}$, and the quartile levels were as follows: quartile $1(<9.8 \mu \mathrm{mol} / \mathrm{L})$, quartile $2(9.8 \mu \mathrm{mol} / \mathrm{L}-12.3 \mu \mathrm{mol} / \mathrm{L})$, quartile $3(12.4 \mu \mathrm{mol} / \mathrm{L}-$ $16.4 \mu \mathrm{mol} / \mathrm{L})$, and quartile $4(>16.4 \mu \mathrm{mol} / \mathrm{L})$. The proportion of hypertension, DM, and previous stroke/TIA was significant greater in the lowest quartile of TBil $(P=0.034,0.039$, and 0.033 respectively; Table 1). Compared with the quartile 4 , the level of C-reaction protein (CRP), total cholesterol (TC), and low density lipoprotein (LDL) were significant higher in patients with the quartile 1 ( $P=0.036,0.026$, and 0.002 respectively; Table 1). There was no significant difference in age, sex, smoking, history of cardiovascular diseases (CVD), initial NIHSS scores, levels of triglyceride (TG), or high density lipoprotein (HDL) among the four groups $(P>0.05)$. Approximately $63(21.6 \%)$ of the patients were diagnosed with END within the first 7 days after stroke onset. The proportion of END was significant higher in the lowest quartile of TBil $(P=0.005$, Table 1). 
Table I Baseline Characteristics of the Study Subjects by Quartiles of Total Bilirubin

\begin{tabular}{|c|c|c|c|c|c|c|c|}
\hline & $\begin{array}{l}\text { Total } \\
(n=291)\end{array}$ & $\begin{array}{c}\text { Quartile I } \\
n=74\end{array}$ & $\begin{array}{c}\text { Quartile } 29.8-12.3 \\
n=72\end{array}$ & $\begin{array}{c}\text { Quartile } 3 \text { I2.4-16.4 } \\
n=73\end{array}$ & $\begin{array}{c}\text { Quartile } 4>16.4 \\
n=72\end{array}$ & $\begin{array}{c}P^{a} \\
\text { value }\end{array}$ & $\begin{array}{c}P^{b} \\
\text { value }\end{array}$ \\
\hline Age, $($ mean $\pm S D)$, years & $69.4 \pm 12.6$ & $67.7 \pm 12.4$ & $68.9 \pm 13.3$ & $69.8 \pm 11.8$ & $71.4 \pm 12.7$ & 0.43 & \\
\hline Male, n (\%) & $160(55.0)$ & $38(51.4)$ & $40(55.6)$ & $4 \mid(56.2)$ & $4 \mid(56.9)$ & 0.91 & \\
\hline Hypertension, n (\%) & $195(67.0)$ & $58(78.4)$ & $51(70.8)$ & $43(58.9)$ & $43(59.7)$ & 0.034 & 0.024 \\
\hline DM, n (\%) & $66(22.7)$ & $23(31.1)$ & $19(26.4)$ & $15(20.5)$ & $9(12.5)$ & 0.039 & 0.012 \\
\hline Smoking, n (\%) & $55(18.9)$ & $13(17.6)$ & $16(22.2)$ & $15(20.5)$ & II (15.3) & 0.72 & \\
\hline Previous stroke/TIA, n (\%) & $51(17.5)$ & $19(25.7)$ & $16(22.2)$ & $8(11.0)$ & $8(11.1)$ & 0.033 & 0.40 \\
\hline CHD, n (\%) & $14(4.8)$ & $5(6.8)$ & $4(5.6)$ & $2(2.7)$ & $3(4.2)$ & 0.74 & \\
\hline Initial NIHSS (median [IQR]) & $3[2-5]$ & $4[1-8]$ & $3[1-6]$ & $2[2-5]$ & $2[1-5]$ & 0.23 & \\
\hline END, n (\%) & $63(21.6)$ & $23(31.1)$ & $18(25.0)$ & $12(16.4)$ & $10(13.9)$ & 0.005 & 0.022 \\
\hline CRP (ug/l) & $7.7 \pm 10.3$ & $12.5 \pm 13.3$ & $8.4 \pm \mid 4.1$ & $6.1 \pm 11.4$ & $3.5 \pm 10.3$ & 0.036 & 0.009 \\
\hline TC (mmol/l) & $4.93 \pm 3.11$ & $6.14 \pm 4.33$ & $5.21 \pm 5.27$ & $4.31 \pm 4.7 \mid$ & $3.87 \pm 5.21$ & 0.026 & 0.011 \\
\hline TG (mmol/l) & $1.53 \pm 1.7 \mid$ & $1.98 \pm 2.53$ & $1.63 \pm 2.12$ & $1.31 \pm 2.26$ & $1.17 \pm 2.35$ & 0.11 & \\
\hline $\mathrm{HDL}(\mathrm{mmol} / \mathrm{l})$ & $1.16 \pm 0.41$ & $1.24 \pm 0.77$ & $1.17 \pm 0.58$ & $1.04 \pm 0.63$ & $1.10 \pm 0.49$ & 0.73 & \\
\hline LDL (mmol/l) & $2.85 \pm 0.82$ & $3.44 \pm 1.76$ & $3.11 \pm 1.63$ & $2.68 \pm 1.59$ & $2.44 \pm 1.66$ & 0.002 & 0.024 \\
\hline
\end{tabular}

Notes: ${ }^{a}$ Comparison between four groups; ${ }^{b}$ Comparison between Quartile I and Quartile 4 if $P^{a}<0.05$. The data are shown as mean \pm SD, median [QI, Q3], or $n$ (\%). Abbreviations: DM, diabetes mellitus; TIA, transient ischemic attack; CHD, coronary heart disease; NIHSS, National Institutes of Health Stroke Scale; IQR, interquartile range; END, early neurological deterioration; CRP, C-Reaction protein; TC, total cholesterol; TG, triglyceride; HDL, high-density lipoprotein cholesterol; LDL, low-density lipoprotein cholesterol; SD, standard deviation.

\section{Baseline Characteristics According to END}

Compared to patients without END, patients with END were older $(P=0.017)$, had a higher proportion of DM $(P<0.001)$, and higher CRP and LDL levels $(P=0.011$ and 0.036 , respectively). The proportion of patients with an elevated TBil level was significantly lower in the END group than in the control (patients without END) group $(P=0.005$; Table 2). There was no significant difference in sex, smoking, history of hypertension, previous stroke/

Table 2 Baseline Characteristics According to Early Neurological Deterioration

\begin{tabular}{|c|c|c|c|}
\hline & END Group $(n=63)$ & Control Group $(n=228)$ & ${ }^{a} P$ value \\
\hline Age, (mean $\pm S D)$, years & $73.5 \pm 14.7$ & $68.1 \pm 10.2$ & 0.017 \\
\hline Male, n (\%) & $30(47.6)$ & $130(57.0)$ & 0.18 \\
\hline Hypertension, n (\%) & $47(74.6)$ & I 48 (64.9) & 0.15 \\
\hline DM, n (\%) & $26(4 I .3)$ & $40(17.5)$ & $<0.001$ \\
\hline Smoking, n (\%) & $13(20.6)$ & $42(18.4)$ & 0.69 \\
\hline Previous stroke/TIA, n (\%) & $12(19.0)$ & $39(17.1)$ & 0.72 \\
\hline CHD, n (\%) & $4(6.3)$ & $10(4.4)$ & 0.53 \\
\hline Initial NIHSS (median [IQR]) & $3[2-7]$ & $3[1-9]$ & 0.64 \\
\hline CRP (ug/l) & $9.8 \pm 5.1$ & $7.1 \pm 1.94$ & 0.011 \\
\hline $\mathrm{TC}(\mathrm{mmol} / \mathrm{l})$ & $5.13 \pm 4.22$ & $4.87 \pm 2.98$ & 0.33 \\
\hline TG (mmol/l) & $1.56 \pm 2.76$ & $1.52 \pm 1.12$ & 0.87 \\
\hline $\mathrm{HDL}(\mathrm{mmol} / \mathrm{l})$ & $1.19 \pm 0.55$ & $1.15 \pm 0.27$ & 0.63 \\
\hline LDL (mmol/l) & $3.18 \pm 1.46$ & $2.76 \pm 0.64$ & 0.036 \\
\hline Quartiles of TBil, $n(\%)$ & & & 0.005 \\
\hline Quartile I & $23(36.5)$ & $51(22.4)$ & \\
\hline Quartile 2 & $18(28.6)$ & $54(23.7)$ & \\
\hline Quartile 3 & $12(19.0)$ & $61(26.8)$ & \\
\hline Quartile 4 & $10(15.9)$ & $62(23.1)$ & \\
\hline
\end{tabular}

Notes: ${ }^{a}$ Comparison between control and END groups. The data are shown as mean \pm SD, median [QI, Q3], or $n$ (\%).

Abbreviations: END, early neurological deterioration; DM, diabetes mellitus; CHD, congestive heart failure; TIA, transient ischemic attack; NIHSS, National Institutes of Health Stroke Scale; IQR, interquartile range; CRP, C-Reaction protein; TC, total cholesterol; TG, triglyceride; HDL, high-density lipoprotein cholesterol; LDL, low-density lipoprotein cholesterol; TBil, total bilirubin; SD, standard deviation. 
TIA, CVD, initial NIHSS scores, TC, TG, or HDL in the two groups $(P>0.05$; Table 2$)$. The risk of END increased with the level of TBil.

\section{Multivariate Analysis According to END TBil Levels}

A decreased level of TBil was associated with an increased risk of END (odds ratio $[\mathrm{OR}]=2.93$ and 1.98 in the first and second quartiles, respectively; $P$ for trend, 0.017; Table 3). After controlling for age, sex, hypertension and DM, the first and second quartiles of TBil were still associated with END $(\mathrm{OR}=1.61$ and 1.46 , respectively; Model 1, $P$ for trend, 0.033; Table 3). After controlling for age, sex, hypertension, DM, NIHSS score, CRP, TC, TG, and LDL, the first quartiles of TBil were still associated with END (OR=1.34; Model 2, $P$ for trend, 0.044; Table 3). In addition, an increased level of CRP and age were also associated with an increased risk of END $(\mathrm{OR}=1.27$ and 1.12 respectively; Table 3$)$.

\section{Discussion}

In this study, we investigated the impact of TBil on END in patients with acute ischemic stroke. We found that the lowest level of TBil was an independently associated with END, which demonstrated that TBil is important in acute ischemic stroke. Compared with patients with the highest level of TBil, the risk of END in patients in quartile 1 increased by 1.38 times. In addition, increased age and level of CRP may be independent predictors of END, independent of established conventional risk factors.

END in acute ischemic stroke is a common event that has been associated with reduced neurological function and an increase in the rate of disability and mortality. ${ }^{1}$ Helleberg's study indicated that the prevalence of END was $13.9 \%$. They defined END as a decrease $\geq 2$ points in any Scandinavian Stroke Scale item for consciousness, gaze, arm and leg motor power, or 3 points for speech. ${ }^{11}$ In a Japanese study, $25 \%$ of acute stroke patients had END. ${ }^{12}$ Our study demonstrated that the incidence of END was 21.6\%. However, END was defined as an increase in the NIHSS score of $\geq 2$ points during the first 7 days of symptom onset in our study. ${ }^{9}$ Previous studies indicated that END prevalence varied from $13 \%$ to $38 \%$ after acute ischemic stroke, possibly due to the different study designs, definition, neurological function assessment methods and source of subjects. ${ }^{2}$

The mechanisms for END in patients with ischemic stroke are not yet completely understood. The occurrence of END is caused by multiple factors, such as age, diabetes, infection, inflammation, metabolic, hemodynamic causes, and seizures. ${ }^{13,14}$ Previous studies suggested that reduced oxygen delivery and regional metabolic disturbances appeared to be the most common fundamental pathophysiological mechanism of END. ${ }^{15,16}$ Bilirubin is the end metabolic production of heme and has been considered as one of the strongest endogenous antioxidant, anti-inflammatory, and neuroprotective molecules as a hormone in mammalian tissues whose actions have been mostly characterized in the high micromolar range. ${ }^{4,17}$ Oda et $\mathrm{al}^{8}$ reported that low TBil was associated with a higher prevalence of stroke in a Japanese health screening population. Another study suggested that higher serum TBil level is associated with improved stroke outcomes. ${ }^{6}$ A meta-analysis of existing studies has confirmed that serum bilirubin concentrations are inversely related to CVD. ${ }^{18}$ Bilirubin, as a hormone, was shown to reduce body weight by reshaping the peroxisome proliferator-activated receptor $\alpha$ (PPAR $\alpha$ ) coregulator profile, remodeling white adipose tissue to improve metabolic function, and reducing plasma TGs, very LDL and fat accumulation which are directly linked to increased stroke. ${ }^{19,20}$ However, the relationship between

Table 3 Multivariate Analysis According to END

\begin{tabular}{|c|c|c|c|c|c|c|}
\hline TBil & Unadjusted OR $(95 \% \mathrm{Cl})$ & $P$ value & Model I & $P$ value & Model 2 & $P$ value \\
\hline$P$ for trend & 0.017 & 0.033 & 0.044 & & & \\
\hline Quartile I & $2.93(1.24-5.77)$ & $0.003^{\mathrm{a}}$ & $1.61(1.23-4.93)$ & $0.019^{\mathrm{a}}$ & $1.34(1.28-3.24)$ & $0.026^{\mathrm{a}}$ \\
\hline Quartile 2 & $1.98(1.42-4.28)$ & $0.021^{\mathrm{a}}$ & $1.46(1.25-3.17)$ & $0.035^{\mathrm{a}}$ & $1.14(0.88-2.46)$ & $0.150^{\mathrm{a}}$ \\
\hline Quartile 3 & $1.47(0.86-2.7 I)$ & $0.060^{\mathrm{a}}$ & $\mathrm{I} .13(0.7 \mathrm{I}-2.93)$ & $0.130^{\mathrm{a}}$ & $1.05(0.67-2.77)$ & $0.320^{\mathrm{a}}$ \\
\hline Quartile 4 & I (reference) & 1 & I & & & \\
\hline Age & $1.76(1.16-4.71)$ & 0.019 & $1.58(1.33-4.44)$ & 0.027 & $1.27(1.07-3.88)$ & 0.044 \\
\hline CRP & $1.53(1.36-3.55)$ & 0.012 & $1.36(1.24-3.58)$ & 0.025 & $1.12(1.05-3.49)$ & 0.040 \\
\hline
\end{tabular}

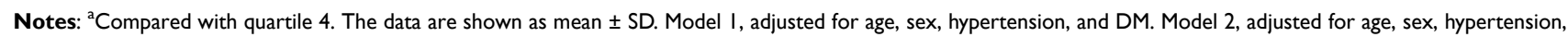
DM, NIHSS score, CRP, TC, TG, and LDL.

Abbreviations: TBil, total bilirubin; CRP, C-Reaction protein; OR, odds ratio; Cl, Confidence interval. 
bilirubin level and END is still controversial, with only a few studies on this topic. One study reported that higher serum bilirubin was correlated with the higher NIHSS scores. ${ }^{7}$ This study explained that severe strokes were associated with a higher bilirubin concentration, which can limit further neurologic damage and is the reason why higher bilirubin levels are associated with better stroke outcomes. Geng et al found that direct bilirubin level at admission, dose not indirect and total bilirubin, was significantly associated with END in acute ischemic stroke. The increase of direct bilirubin levels may be related to END. ${ }^{21}$ Another study indicated that the level of indirect bilirubin was significantly decreased in the acute phase of stroke. ${ }^{22}$ In our study, patients with END had a incidence of DM and higher level of LDL and CRP, which were negatively associated with the TBil level. After adjustment for potential variables, the first quartile of TBil was associated with END. These results suggested that the lowest level of TBil is a possible predictor for the development of END.

Oxidized LDL has been known to be involved in the process of atherosclerosis. ${ }^{23}$ Numerous studies have shown a negative relationship between serum bilirubin levels and atherosclerosis. ${ }^{24-26}$ A meta-analysis reported a close negative relationship was found between ordered serum bilirubin levels and severity of atherosclerosis. ${ }^{27}$ Oda reported that bilirubin suppresses the oxidation of lipid in liposomes, which is regarded as the best antioxidant of lipid peroxidation. ${ }^{28}$ In our study, compared with patients with the highest TBil levels, patients with the lowest TBil levels had significantly higher LDL levels. On the other hand, we found that patients with END had decreased TBil levels and increased LDL levels. Therefore, bilirubin may be able to inhibit oxidant damage related to atherosclerosis disease.

Inflammation plays a key role in the pathogenesis of stroke. CRP has been regarded as a non-specific marker of the acute response to inflammation, infection, and tissue injury, and is the most extensively used and established marker to date. ${ }^{29}$ A recent study has shown that an increase in CRP is positively associated with an increased risk and severity of stroke. ${ }^{30}$ Another previous study demonstrated that high concentrations of inflammatory markers in the blood are associated with END. ${ }^{31}$ Growing evidence suggests that bilirubin could decrease inflammatory responses by preventing the migration of

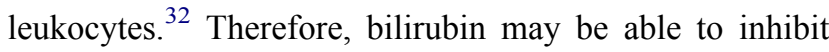
neuroinflammation-related END.
There are some limitations to our study. First, this study was a retrospective single-center study with a small sample size, and the results needs further verification in a larger, prospective, multi-center study. Second, we measured bilirubin at admission and the level of bilirubin in patients had not been followed up. Third, different definition of END may have resulted in bias in the evaluation of END. Finally, stroke subtypes had not been classified. However, the bilirubin was not various with the Chinese ischemic stroke subclassification. ${ }^{12}$

\section{Conclusion}

In conclusion, END after acute ischemic stroke is likely a multifactorial event. Our study shows a negative relationship between TBil levels at admission and END in patients with acute ischemic stroke. It suggest that TBil level in patients with acute cerebral infarction may be a useful biomarker for the prediction of END. In the future, prospective studies are needed to clarify the role of bilirubin in the END.

\section{Ethical Approval}

The study was approved by the Ethical Committee of Suzhou Ninth People's Hospital (No. WJYY-201747) and conducted in accordance with the Declaration of Helsinki.

\section{Funding}

This work was supported by the Suzhou Youth Science and technology project (KJXW2016065) and Basic research of Suzhou Medical and health care (SYSD2020044).

\section{Disclosure}

The authors declared no potential conflicts of interest with respect to the research, authorship, and/or publication of this article.

\section{References}

1. Seners P, Turc G, Oppenheim C, et al. Incidence, causes and predictors of neurological deterioration occurring within $24 \mathrm{~h}$ following acute ischaemic stroke: a systematic review with pathophysiological implications. J Neurol Neurosurg Psychiatry. 2015;86(1):87-94. doi:10.1136/jnnp-2014-308327

2. Kim TJ, Kang IK, Jeong H, et al. Cystatin $\mathrm{C}$ is a useful predictor of early neurological deterioration following ischaemic stroke in elderly patients with normal renal function. Eur Stroke J. 2017;2(1):23-30. doi: $10.1177 / 2396987316677197$

3. Martin AJ, Price CI. A systematic review and meta-analysis of molecular biomarkers associated with early neurological deterioration following acute stroke. Cerebrovasc Dis. 2018;46(5-6):230-241. doi: $10.1159 / 000495572$ 
4. Thakkar M, Edelenbos J, Doré S. Bilirubin and ischemic stroke: rendering the current paradigm to better understand the protective effects of bilirubin. Mol Neurobiol. 2019;56(8):5483-5496. doi:10.1007/s12035-018-1440-y

5. Huang YH, Yang YC, Lu FH, et al. Serum bilirubin is inversely associated with increased arterial stiffness in men with pre-hypertension but not normotension. PLoS One. 2016;11(1): e146226.

6. Perlstein TS, Pande RL, Creager MA, et al. Serum total bilirubin level, prevalent stroke, and stroke outcomes: national health and nutrition examination survey. Am J Med. 2008;121(9):781-788. doi:10.1016/j.amjmed.2008.03.045

7. Luo Y, Li JW, Lu ZJ, et al. Serum bilirubin after acute ischemic stroke is associated with stroke severity. Curr Neurovasc Res. 2012;9 (2):128-132. doi:10.2174/156720212800410876

8. Oda E, Oda E, Kawai R, et al. A possible cross-sectional association of serum total bilirubin with coronary heart disease and stroke in a Japanese health screening population. Heart Vessels. 2012;27 (1):29-36. doi:10.1007/s00380-011-0123-7

9. Jeong-Min K, Bae J-H, Park K-Y. Incidence and mechanism of early neurological deterioration after endovascular thrombectomy. J Neurol. 2019;266(3):609-615. doi:10.1007/s00415-018-09173-0

10. Duan Z, Shan W, Du H, et al. Association between serum retinoic acid levels and risk of post-stroke depression in patients with ischemic stroke. Asian J Psychiatr. 2019;46:87-91. doi:10.1016/j. ajp.2019.09.038

11. Helleberg BH, Ellekjaer H, Indredavik B. Outcomes after early neurological deterioration and transitory deterioration in acute ischemic stroke patients. Cerebrovasc Dis. 2016;42(5):378-386. doi: $10.1159 / 000447130$

12. Tei H, Uchiyama S, Ohara K, et al. Deteriorating ischemic stroke in 4 clinical categories classified by the oxfordshire community stroke project. Stroke. 2000;31(9):2049-2054. doi:10.1161/01. STR.31.9.2049

13. Vila N, Castillo J, Dávalos A, et al. Proinflammatory cytokines and early neurological worsening in ischemic stroke. Stroke. 2000;31 (10):2325-2329. doi:10.1161/01.STR.31.10.2325

14. Chung JW, Kim N, Kang J, et al. Blood pressure variability and the development of early neurological deterioration following acute ischemic stroke. J Hypertens. 2015;33(10):2099-2106. doi:10.1097/ HJH.0000000000000675

15. Caplan RL. Worsening in ischemic stroke patients: is it time for a new strategy? Stroke. 2002;33(6):1443-1445. doi:10.1161/01. STR.0000016924.55448.43

16. Carbonell T, Rama R. Iron, oxidative stress and early neurological deterioration in ischemic stroke. Curr Med Chem. 2007;14 (8):857-874. doi:10.2174/092986707780363014

17. Creeden JF, Gordon DM, Stec DE, et al. Bilirubin as a metabolic hormone: the physiological relevance of low levels. Am J Physiol Endocrinol Metab. 2021;320(2):E191-E207. doi:10.1152/ ajpendo.00405.2020

18. Novotny L, Vítek L. Inverse relationship between serum bilirubin and atherosclerosis in men: a meta-analysis of published studies. Exp Biol Med. 2003;228(5):568-571. doi:10.1177/15353702-0322805-29

Neuropsychiatric Disease and Treatment

\section{Publish your work in this journal}

Neuropsychiatric Disease and Treatment is an international, peerreviewed journal of clinical therapeutics and pharmacology focusing on concise rapid reporting of clinical or pre-clinical studies on a range of neuropsychiatric and neurological disorders. This journal is indexed on PubMed Central, the 'PsycINFO' database and CAS, and
19. Gordon DM, Neifer KL, Hamoud AA, et al. Bilirubin remodels murine white adipose tissue by reshaping mitochondrial activity and the coregulator profile of peroxisome proliferator-activated receptor alpha. J Biol Chem. 2020;295(29):9804-9822. doi:10.1074/jbc. RA120.013700

20. Hinds TJ, Creeden JF, Gordon DM, et al. Bilirubin nanoparticles reduce diet-induced hepatic steatosis, improve fat utilization, and increase plasma beta-hydroxybutyrate. Front Pharmacol. 2020;11:594574. doi:10.3389/fphar.2020.594574

21. Geng HH, Wang Q, Li B, et al. Early neurological deterioration during the acute phase as a predictor of long-term outcome after first-ever ischemic stroke. Medicine (Baltimore). 2017;96(51): e9068. doi:10.1097/MD.0000000000009068

22. Muscari A, Collini A, Fabbri E, et al. Changes of liver enzymes and bilirubin during ischemic stroke: mechanisms and possible significance. BMC Neurol. 2014;14:122. doi:10.1186/1471-2377-14122

23. Tavridou A, Efthimiadis A, Efthimiadis I, et al. Simvastatin-induced changes in circulating oxidized low-density lipoprotein in different types of dyslipidemia. Heart Vessels. 2010;25(4):288-293. doi:10.1007/s00380-009-1202-x

24. Vítek L. Bilirubin and atherosclerotic diseases. Physiol Res. 2017;66 (Supplementum1):S11-S20. doi:10.33549/physiolres.933581

25. Zhong K, Wang X, Ma X, et al. Association between serum bilirubin and asymptomatic intracranial atherosclerosis: results from a Population-Based Study. Neurol Sci. 2020;41(6):1531-1538. doi:10.1007/s10072-020-04268-x

26. Yan W. Association of serum neuron-specific enolase and bilirubin levels with cerebral dysfunction and prognosis in large-artery atherosclerotic strokes. J Cell Biochem. 2019.

27. Lan Y, Liu H, Liu J, et al. Is serum total bilirubin a predictor of prognosis in arteriosclerotic cardiovascular disease? A meta-analysis. Medicine. 2019;98(42):e17544. doi:10.1097/MD.0000000000017544

28. Oda E. A decrease in total bilirubin predicted hyper-LDL cholesterolemia in a health screening population. Atherosclerosis. 2014;235 (2):334-338. doi:10.1016/j.atherosclerosis.2014.05.927

29. Sproston NR, Ashworth JJ. Role of C-reactive protein at sites of inflammation and infection. Front Immunol. 2018;9:754. doi:10.3389/fimmu.2018.00754

30. Luna JM, Moon YP, Liu KM, et al. High-sensitivity C-reactive protein and interleukin-6-dominant inflammation and ischemic stroke risk: the Northern Manhattan Study. Stroke. 2014;45(4):979-987. doi:10.1161/STROKEAHA.113.002289

31. Castellanos M, Castillo J, García MM, et al. Inflammation-mediated damage in progressing lacunar infarctions a potential therapeutic target. Stroke. 2002;33(4):982-987. doi:10.1161/hs0402.105339

32. Keshavan P, Deem TL, Schwemberger SJ, et al. Unconjugated bilirubin inhibits VCAM-1-mediated transendothelial leukocyte migration. $J$ Immunol. 2005;174(6):3709-3718. doi:10.4049/ jimmunol.174.6.3709 is the official journal of The International Neuropsychiatric Association (INA). The manuscript management system is completely online and includes a very quick and fair peer-review system which is all easy to use. Visit http://www.dovepress.com/testimonials.php to read real quotes from published authors.

\section{Dovepress}

